\title{
Penicillin allergy: anti-penicillin IgE antibodies and immediate hypersensitivity skin reactions employing major and minor determinants of penicillin
}

\author{
R K CHANDRA, S A JOGLEKAR, AND E TOMAS \\ Department of Paediatrics, Memorial University of Newfoundland, and Dr Charles A Janeway \\ Child Health Centre, St John's, Newfoundland, Canada
}

SUMMARY 300 children considered to have had adverse reactions to penicillin were examined. Informed consent was obtained from the parents. Skin tests were conducted by the scratch/prick and intradermal techniques, using benzylpenicilloyl polylysine conjugate and a mixture of minor determinants of penicillin. Specific anti-penicillin IgE antibodies were estimated by the radioallergosorbent test. There was a good correlation between the two methods. The overall frequency of positive tests was $19 \%$. 11 children showed cutaneous reactivity only to the minor determinants mixture. Positive results were found more often in those with accelerated adverse reactions, particularly anaphylaxis, serum sickness, angio-oedema, or urticaria. The validity of penicillin-negative results was confirmed by drug challenge in 56 subjects, only 2 of whom showed a slight skin rash. Of 5 patients with positive tests, inadvertent administration of penicillin produced accelerated urticaria in all. 14 of 42 children with positive tests had lost hypersensitivity to penicillin one year later. In a separate group of 50 children with a history of adverse response to ampicillin, the overall frequency of positive tests was $12 \% ; 38 \%$ showed evidence of recent E-B virus infection. It was concluded that penicillin allergy is often overdiagnosed. The diagnosis can be reliably confirmed by skin tests using major and minor determinants of benzylpenicillin and by the radioallergosorbent test; such hypersensitivity is not permanent.

Allergy to penicillin is the most frequently diagnosed drug adverse reaction. In our hospital, $8 \%$ of all patients are alleged to have hypersensitivity to penicillin. Since the penicillin group of antibiotics is the safest and most effective antibacterial, it is desirable to find out whether a patient is allergic to the drug. Apart from clinical history, there are two methods for confirming or excluding the diagnosis: (1) Skin tests using major and minor determinants of penicillin*. ${ }^{1-3}$ (2) Penicilloyl-specific IgE anti-

*The generic term penicillin is used for the entire group of natural and semisynthetic penicillins. The basic structure of the compound consists of a thiazolidine ring connected to a beta-lactam ring to which is attached a side chain. The integrity of the penicillin nucleus, 6-aminopenicillanic acid, is essential for biological activity. Penicillinase breaks down the molecule to penicilloic acids. Hypersensitivity can occur to the 'major determinant' penicilloyl polylysine (called 'major' because of the frequency with which hyperactivity to this compound underlies allergic reactions to penicillin), to the 'minor determinants' (metabolic degradation products of penicillin; these are called 'minor' because of the relative infrequency of allergy to these compounds, although the severity of adverse reaction can vary from slight to fatal), or to both. For further information on immunochemistry of penicillin hypersensitivity, see Clarke et al..$^{5}$ bodies measured by the radioallergosorbent test (RAST). ${ }^{4}$ We have investigated a large series of children using both these methods and find that penicillin allergy is frequently overdiagnosed; only $19 \%$ of them showed positive tests.

\section{Subjects and methods}

300 children attending the hospital as outpatients or inpatients were referred to one of us (RKC) for evaluation of suspected allergy to penicillin. An additional 50 children with alleged allergy to ampicillin were tested separately. Any child who was known to have had penicillin without any adverse reaction since the earlier diagnosis of penicillin hypersensitivity was excluded. Informed consent was obtained from the parents. The study protocol had been approved by the Human Experimentation Committees of the hospital and the university.

A detailed history of the suspected adverse reaction to penicillins was obtained from the parents; this was supplemented by information obtained from the attending physician or hospital records, or both. The 
indications for treatment with penicillin were upper respiratory infection (in 153), otitis (101), sore throat (54), pneumonia (26), urinary infection (18), and skin infection (12). The following reagents were used for skin tests: benzylpenicilloyl-polylysine (KremersUrban) in a concentration of $6 \times 10^{-5} \mathrm{~mol} / \mathrm{l}$, minor determinant mixture (MDM) containing sodium benzylpenicillin G, sodium D-benzylpenicilloate, and sodium D-benzylpenilloate each in a concentration of $1 \times 10^{-2} \mathrm{~mol} / \mathrm{l}$. In some children, MDM of ampicillin was also used. All test reagents were dissolved in sterile $0.01 \mathrm{~mol} / \mathrm{l}$ phosphate buffer and $0 \cdot 15 \mathrm{~mol} / \mathrm{l}$ sodium chloride. Phosphate-buffered saline was used as a negative control and to rule out skin reactions due to dermatographism.

Skin testing was done on the inner volar surface of the forearm. In each child, a scratch or prick test was performed initially. If there was no reaction to the scratch or prick test within 10 minutes, an intradermal test was performed by injecting $0.03 \mathrm{ml}$ of the material intracutaneously with a 26 gauge needle on a tuberculin syringe. The patients were observed for at least 20 minutes. A weal reaction of $3 \mathrm{~mm}$ larger than that of the saline control was considered to be positive. Skin tests were deferred if the adverse reaction to penicillin had taken place within the previous month, or if the patient had received antihistamines or corticosteroids within the preceding 48 hours.

Blood was obtained from each patient and the serum stored at $-20^{\circ} \mathrm{C}$ for up to 4 weeks until the in vitro tests were performed. Immunoglobulins were estimated immunochemically by the method of single radial diffusion in agar or nephelometry using monospecific antiserum (Hyland). IgE was estimated by radioimmunoassay (Pharmacia). RAST was performed using benzylpenicilloyl and phenoxymethylpenicilloyl conjugated to human serum albumin, and coupled to cyanogen bromideactivated cellulose paper discs (Pharmacia).

\section{Results}

The overall frequency of positive tests-skin reaction, RAST, or both-was $19 \%$ (Table 1). There was a

Table 1 Incidence of positive tests for penicillin allergy

$\left.\begin{array}{lcc}\hline \text { Procedure } & \multicolumn{2}{c}{\text { Children with positive tests }} \\ \hline \text { Skin tests } & 30 & 48(16)^{*} \\ \quad \text { Major determinant } & 11 & \\ \quad \text { Minor determinants } & 7 & \\ \quad \text { Both } & & 42(14) \\ \text { RAST } & & 33(11) \\ \text { Skin tests + RAST } & & 9(5) \\ \text { Skin tests only } & & 9(3)\end{array}\right\} 57(19)$

*Percentage of the total number of 300 children. significant correlation between immediate hypersensitivity skin tests and the in vitro technique for estimating IgE antibodies to penicillin, both were positive or negative in $78 \%$ of patients. 11 children showed a positive reaction only to MDM. Positive results were obtained more often in those who had recently been treated with penicillin (Table 2), in those with an accelerated adverse reaction (Table 3), and if the hypersensitivity manifested as anaphylaxis, angio-oedema, serum sickness, or urticaria (Table 4). Total serum IgE was raised in 42 subjects but this did not correlate with the presence of antipenicillinspecific IgE antibodies or with positive skin tests. Many individuals with total serum IgE within the normal range had evidence of penicillin hypersensitivity as judged by RAST or cutaneous reactions.

The validity of the testing procedures was shown by subsequent drug challenge in 56 children with negative tests; only 2 showed a slight erythematous, non-itchy skin rash. The inadvertent administration

Table 2 Relationship of positive results to time elapsed since adverse reaction

\begin{tabular}{lc}
\hline Time interval (months) & $\begin{array}{l}\text { Percentage of children with } \\
\text { positive results }\end{array}$ \\
\hline $1-3$ & $18 \cdot 6$ \\
$4-12$ & $9 \cdot 3$ \\
$13-60$ & $4 \cdot 5$ \\
$>60$ & $1 \cdot 9$ \\
\hline
\end{tabular}

Table 3 Relationship of positive results to speed of adverse reaction

\begin{tabular}{ll}
\hline $\begin{array}{l}\text { Duration of treatment with } \\
\text { penicillin before adverse reaction } \\
\text { (hours) }\end{array}$ & $\begin{array}{l}\text { Percentage of children with } \\
\text { positive results }\end{array}$ \\
\hline$<12$ & $21 \cdot 2$ \\
$13-24$ & $11 \cdot 9$ \\
$25-48$ & $6 \cdot 3$ \\
$49-72$ & $2 \cdot 1$ \\
$>72$ & $1 \cdot 9$ \\
\hline
\end{tabular}

Table 4 Relationship of positive results to the nature of adverse reaction

\begin{tabular}{lrcc}
\hline Manifestation & No tested & $\begin{array}{l}\text { Children with positive } \\
\text { results }\end{array}$ \\
\cline { 3 - 4 } & & No & $\%$ \\
\hline Accelerated skin rash* & 68 & 17 & 25 \\
Delayed skin rash $\dagger$ & 159 & 9 & $5 \cdot 6$ \\
Urticaria & 49 & 18 & $36 \cdot 7$ \\
Angio-oedema & 15 & 8 & $54 \cdot 4$ \\
Serum sickness & 2 & 2 & 100 \\
Anaphylaxis & 3 & 3 & 100 \\
Others & 4 & - & -
\end{tabular}

*Skin rash appearing within 24 hours of starting treatment with penicillin.†Skin rash observed more than 24 hours after starting treatment with penicillin. 
of penicillin in 5 patients with positive tests resulted in rapid generalised urticaria in each (Table 5).

In 42 children with positive tests, the procedures were repeated one year later. 14 showed negative responses at the time of rechallenge and were given penicillin without any adverse reaction.

The data on 50 children with adverse response to ampicillin were analysed separately (Table 6). It was found that $38 \%$ of children who had developed a maculopapular skin rash after the administration of ampicillin had evidence of past Epstein-Barr virus infection, as shown by positive mono spot test or by immunofluorescent demonstration of antibody to E-B virus. The use of MDM ampicillin did not increase the number of positive tests.

The various allergic and immunological disorders incidentally found in the study group are shown in Table 7.

Table 5 Drug challenge

\begin{tabular}{lllll}
\hline $\begin{array}{l}\text { Total } \\
\text { no }\end{array}$ & $\begin{array}{l}\text { Skin } \\
\text { tests }\end{array}$ & RAST & \multicolumn{2}{c}{ Children with adverse reaction } \\
\cline { 3 - 5 } & & No & Clinical features \\
\hline 56 & Negative & Negative & 2 & $\begin{array}{c}\text { Erythematous non-itchy skin } \\
\text { rash } \\
\text { Accelerated urticaria, one with } \\
\text { angio-oedema }\end{array}$ \\
3 & Positive & Positive & 3 & $\begin{array}{l}\text { Acceleratcd urticaria } \\
\text { Accelerated urticaria }\end{array}$ \\
\hline
\end{tabular}

*This child had reacted only to MDM.

Table 6 Incidence of positive tests in children with adverse reaction to ampicillin

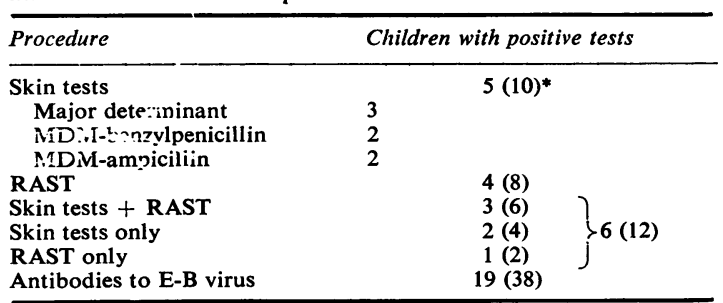

* Percentage of the total number of 50 children.

Two children reacted to MDM-benzylpenicillin as well as to MDMampicillin.

Table 7 Associated allergic and immunological disorders

\begin{tabular}{lr}
\hline Disorder & No $(\%)$ \\
\hline Eczema & $27(7 \cdot 7)^{*}$ \\
Asthma & $26(7 \cdot 4)$ \\
Rhinitis & $19(5 \cdot 4)$ \\
Urticaria & $17(4 \cdot 8)$ \\
Allergy to other drugs & $9(2 \cdot 5)$ \\
Common varied immunodeficiency $16(4 \cdot 5)$ \\
Selective IgA deficiency & $7(2 \cdot 0)$ \\
\hline
\end{tabular}

*Percentage of the total number of 350 children. Some children had more than one clinical manifestation.

\section{Discussion}

Estimates of the incidence of allergy to penicillins range from 1 to $10 \%$ of all patients given this antibiotic. In addition to its extensive use in medicine, there is constant human exposure to penicillin from contamination of foods and from penicillinproducing ubiquitous organisms in the environment. Many different degradation products of penicillin are implicated in penicillin allergy. The most frequently incriminated is the penicilloyl group, the so-called 'major determinant'. It should be stressed however, that reactivity to degradation products of penicillin, the so called 'minor determinants', may be severe. There is the suggestion that MDM reactivity is more often associated with severe anaphylactic reactions. In our study group, the diagnosis of penicillin hypersensitivity might have been missed in 11 patients if MDM had not been included as a test substance. There was a significant correlation between skin reactions and RAST, both tests were positive or negative in $78 \%$ of patients. In another report, skin tests performed with penicilloylpolylysine showed an overall correlation of $91.5 \%$ with RAST results. ${ }^{6}$

In this study, several clinical correlates of obtaining positive tests were observed. Positive tests were seen more often in those with a recent history of drug adverse reaction. Moreover, of 42 children with positive tests who were checked again a year later, 14 had lost their reactivity to penicillin. This may reflect the progressive decline in the titre of penicillinspecific IgE antibodies with time. In some studies, the frequency of positive skin reactivity had declined greatly when the tests were done several years later. Wide and Jublin ${ }^{7}$ obtained positive skin tests and anti-penicillin IgE antibodies in all of 11 patients with a recent episode of anaphylaxis or urticaria after treatment with penicillin but in none of the 12 patients with similar reactions 7 months to 14 years before testing. In another study, the frequency of positive skin reactions decreased from $77 \%$ at 3 months after the adverse reaction, to $25 \%$ after 5 years. ${ }^{8}$ On the other hand, the American Academy of Allergy Study Group ${ }^{9}$ found a lack of strong correlation between the results of skin testing and the interval of time since the earlier penicillin reaction. We did not find any correlation between age and the frequency of positive reactions.

We observed that penicillin hypersensitivity tests were more often positive in individuals with a history of accelerated adverse reactions occurring within 24 hours of starting treatment with penicillin. Delayed reactions are likely to be mediated by immunological mechanisms other than IgE-related hypersensitivity. These symptoms include frequently 
encountered polymorphic skin rashes, including erythema multiforme, and maculopapular and erythematous rashes.

The relatively small number of positive responses obtained in this study may be due to a number of factors. In some patients, tests were performed several months to 7 years after the suspected penicillin adverse reaction. Penicillin-specific IgE antibodies could have declined to a level insufficient to produce a skin reaction or to be detected by RAST. However, it is not established whether further exposure to penicillin might once again induce clinically relevant hypersensitivity. The most common adverse reaction was a skin rash, a symptom with the least yield in terms of positive skin tests or RAST, It is quite probable that many of these skin rashes, particularly maculopapular and polymorphic forms, are integral components of the clinical features of illness for which penicillin was administered. Skin rashes commonly occur in the course of various viral or streptococcal infections. Similarly, vague gastrointestinal symptoms are most unlikely to be due to penicillin hypersensitivity. This is particularly relevant for alleged reactions to ampicillin. In patients with infectious mononucleosis or subclinical E-B virus infection, treatment with ampicillin is often associated with skin rashes. We found evidence of previous E-B virus infection in $38 \%$ of patients with adverse reactions to ampicillin. Moreover, our data do not support a compelling need for the use of antigenic determinants other than those derived from benzylpenicillin for cutaneous reactivity. None of the patients showed greater hypersensitivity to ampicillin or its derivatives than to products prepared from benzylpenicillin.

The frequency of occurrence of other allergic disorders and of common varied immunodeficiency and selective $\operatorname{IgA}$ deficiency in the group was somewhat higher than might be expected in an unselected general population, although the true incidence of these states in various communities is not known. ${ }^{10-12}$ Moreover, the occurrence of allergic disease is related to patterns of infant feeding. ${ }^{13-15}$ It may reflect a fortuitous selection; alternatively IgEmediated allergy to drugs may develop more often in atopic individuals and in those with immunodeficiency. On the other hand, Kraft et al. ${ }^{6}$ did not note the presence or history of atopic disease in any of the patients with proved penicillin allergy.

We conclude that penicillin allergy is frequently overdiagnosed and that such hypersensitivity is not necessarily permanent. The diagnosis of clinically significant allergy to penicillins can be reliably excluded on the basis of both skin tests using major and minor determinants of benzylpenicillin and RAST.
We thank Drs C Hobeika, D Simms, W Sprague, D Hillman, E Hillman, S Chakrabarty, R Dominic, D Vaze, and many other colleagues for referring patients, Dr N Franklin Adkinson for technical information on the preparation of skin testing reagents, and Greg Woodford, Joan Dwyer, and Bing Au for excellent technical support.

Pharmacia, Montreal partly financed this study.

This paper was presented to the Canadian Paediatric Society, Halifax, 20-22 June 1978.

\section{References}

1 Rosenblum A H. Penicillin allergy. J Allergy Clin Immunol 1968 ; 42: 309-18.

2 Levine B B, Zolov D M. Prediction of penicillin allergy by immunological tests. J Allergy Clin Immunol 1969; 43: 231-44.

3 Adkinson N F, Jr, Thompson W L, Maddrey W C, Lichtenstein L M. Routine use of penicillin skin testing on an inpatient service. $N$ Engl J Med 1971; 285: $22-4$.

4 Wide L. Clinical significance of measurement of reaginic (IgE) antibody by RAST. Clin Allergy 1973; 3: 583-95.

5 Clarke H T, Johnson J R, Robinson R, eds. The chemistry of penicillin. Princeton: Princeton University Press, 1949: 563-73.

6 Kraft D, Roth A, Mischer P, Pichler H, Ebner H. Specific and total serum IgE measurements in the diagnosis of penicillin allergy. A long term follow-up study. Clin Allergy 1977; $7: 21-8$.

7 Wide L, Jublin L. Detection of penicillin allergy of the immediate type by radioimmunoassay of reagins (IgE) to penicilloyl conjugates. Clin Allergy 1971; 1: 171-7.

8 Finke S R, Grieco M H, Connell J T, Smith E C, Sherman W B. Results of comparative skin tests with penicilloylpolylysine and penicillin in patients with penicillin allergy. Am J Med 1965; 38: 71-8.

9 Green G R, Rosenblum A. Report of the Penicillin Study Group-American Academy of Allergy. J Allergy Clin Immunol 1971; 48: 331-43.

10 Soothill J F. Immunity deficiency states. In: Clinical aspects of immunology. Gell $\mathrm{P} G \mathrm{H}$, Coombs $\mathrm{R} R \mathrm{~A}$, Lackman P J, eds. Oxford: Blackwell Scientific, 1975: 649-87.

11 Chandra R K, Ghai O P. Primary immunodeficiency states in Indian children. Indian J Med Res 1976; 64: 68-75.

12 World Health Organisation. Immunodeficiency. Technical Report Series No 630. Geneva: WHO, 1978.

13 Mathew D J, Taylor B, Norman A P, Turner M W, Soothill J F. Prevention of eczema. Lancet 1977; i: 321-5.

14 Chandra R K. Breast feeding and prevention of atopic eczema (abstract). Pediatr Res 1978; 12: 478.

15 Chandra R K. Prospective studies of the effect of breast feeding on incidence of infection and allergy. Acta Paediatr Scand 1979; 68: 691-4.

Correspondence to Professor $\mathbf{R} \mathrm{K}$ Chandra, Clinical Research Center, Massachusetts Institute of Technology, 50 Ames Street, Cambridge, Massachusetts 02142, USA.

Received 18 September 1979 